Revue de l'Institut des langues et cultures

d'Europe, Amérique, Afrique, Asie et Australie

37 | 2019

Des genres en Méditerranée : pratiques,

représentations et transfert

\title{
L'énergie dissensuelle du street-art au Maroc. Pouvoirs, politique et poétique d'une pratique artistique urbaine en Méditerranée
}

The Dissension Energy of Street-art in Morocco.

Powers, Politics and Poetics of a Mediterranean Artistic Practice

\section{Cléo Marmié}

\section{OpenEdition \\ Journals}

Édition électronique

URL : http://journals.openedition.org/ilcea/7538

DOI : 10.4000/ilcea.7538

ISSN : 2101-0609

Éditeur

UGA Éditions/Université Grenoble Alpes

\section{Édition imprimée}

ISBN : 978-2-37747-099-0

ISSN : $1639-6073$

Référence électronique

Cléo Marmié, «L'énergie dissensuelle du street-art au Maroc. Pouvoirs, politique et poétique d'une pratique artistique urbaine en Méditerranée », ILCEA [En ligne], 37 | 2019, mis en ligne le 04 novembre 2019, consulté le 15 janvier 2020. URL : http://journals.openedition.org/ilcea/7538 ; DOI : 10.4000/ ilcea.7538

Ce document a été généré automatiquement le 15 janvier 2020.

(c) ILCEA 


\title{
L'énergie dissensuelle du street-art au Maroc. Pouvoirs, politique et poétique d'une pratique artistique urbaine en Méditerranée
}

\author{
The Dissension Energy of Street-art in Morocco. \\ Powers, Politics and Poetics of a Mediterranean Artistic Practice
}

Cléo Marmié

\section{Introduction}

1 En février 2016, l'artiste italien RUN réalise une œuvre magistrale et éphémère qui s'étend sur les $6400 \mathrm{~m}^{2}$ de la place Moulay Hassan d'Essaouira: la plus grande fresque d'Afrique du Nord vient de voir le jour. Elle témoigne non seulement de l'intrusion spectaculaire de l'art dans la rue marocaine, mais également de la célébration institutionnelle et de la reconnaissance internationale du street-art au Maroc.

2 Aussi appelé art public, art urbain ou art de rue, le street-art comprend toute œuvre légale ou illégale, installée, réalisée ou affichée dans l'espace public, ouverte à tou-te-s, dans l'intention déclarée de stimuler et d'enrichir le lieu dans lequel elle s'inscrit. Il recouvre, sur un mode non-exhaustif, le tag, le graffiti, le poster, le détournement publicitaire, le pochoir, la sérigraphie, le sticker, l'installation, mais aussi, dans sa définition élargie, le théâtre de rue, la musique urbaine, la flashmob ou encore le cirque de rue. Le street-art résulte ainsi d'un faisceau de conditions complexes et mouvantes, profondément imprégnées de l'environnement politique, social et culturel dans lequel il intervient. Il a d'ailleurs été un relai particulièrement actif des contestations qui ont embrasé les rues de monde arabe en 2011. De l'Égypte au Bahreïn, la révolte se lisait aussi sur les murs : graffitis, autocollants et tags se sont faits des auxiliaires visuels des revendications des manifestants. Pourtant, les murs du royaume chérifien se sont 
particulièrement distingués par leur mutisme. Alors que la scène du street-art se structurait de manière remarquable au Maroc, elle ne s'est qu'à de rares exceptions près saisie des revendications du Mouvement du 20 Février (M20F). Et, alors que le régime politique marocain est qualifié par les politistes de semi-autoritaire, sa capitale, Rabat, a récemment été classée par le site spécialisé Artsy parmi les meilleures villes du monde pour être street-artiste aujourd'hui. Cela revient-il à considérer le street-art au Maroc comme un "signifiant vide", déserté d'une dimension politique? Or, si les œuvres qui se donnent à voir sur les murs de la rue marocaine ne sont pas explicitement contestataires, elles se révèlent riches en sémiotisation esthétique et culturelle et font montre d'un potentiel dissensuel particulièrement fécond. C'est ce paradoxe, angle mort de la littérature sociologique et des analyses des formes du nonconsentement et de la résistance au Maghreb, que notre réflexion prétend constituer en objet d'étude.

3 Comment les œuvres se saisissent-elles des problématiques socioculturelles et politiques propres à la société marocaine et les donnent-elles à voir ? De quelle manière le street-art au Maroc, qui fait « acte poétique » dans l'espace urbain, peut-il aussi faire acte politique ? Dans quelle mesure cette pratique artistique hors-les-musées, à travers des jeux de résistances et de reconnaissance, interroge-t-elle les rapports de pouvoir, d'hégémonie et de domination?

4 À travers l'étude des modalités par lesquelles le street-art joue avec les frontières du tolérable, du légitime et du colonial, notre réflexion propose ainsi d'explorer la conflictualité et les enjeux de pouvoir d'une pratique artistique inédite au Maroc.

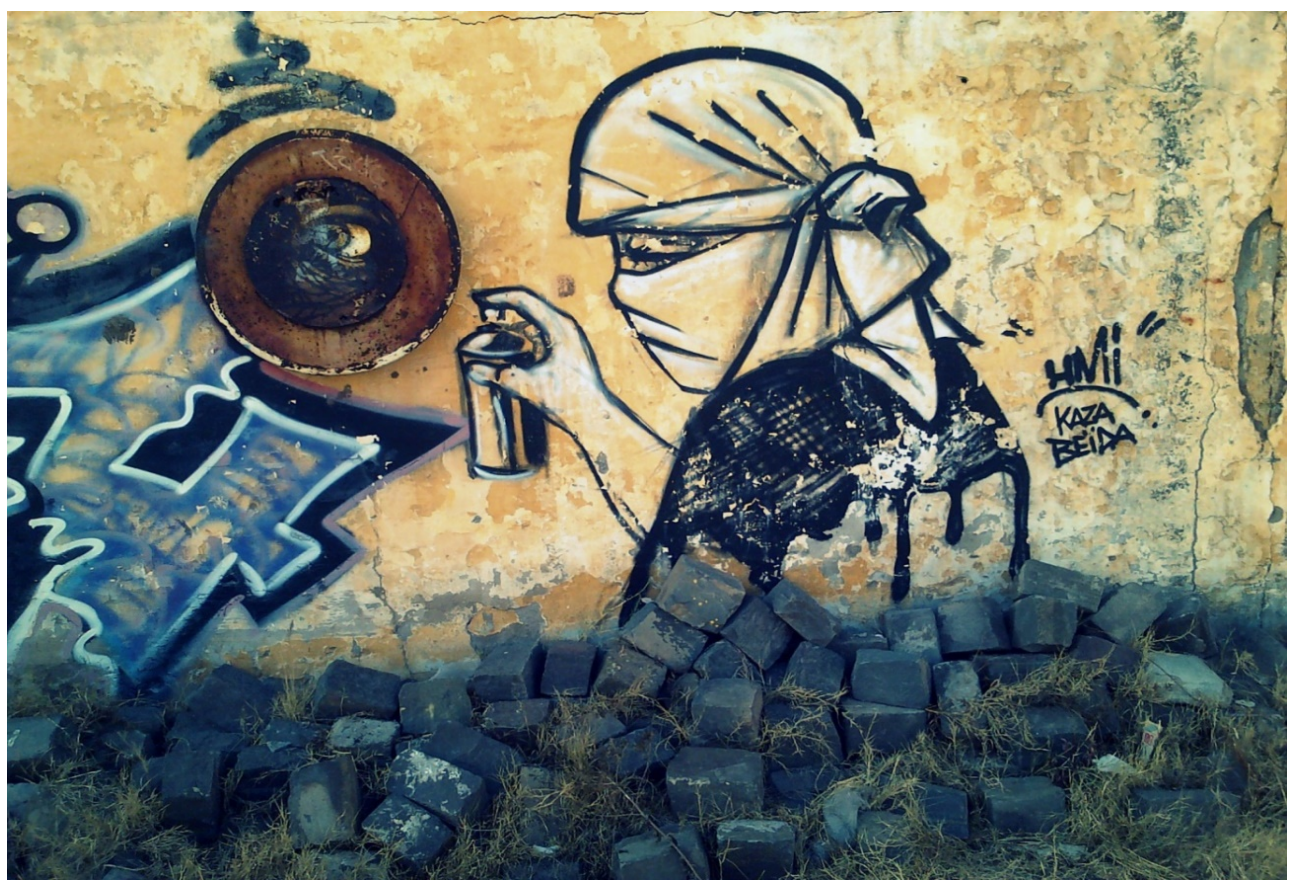

(c) Cléo Marmié

\section{Frontières du tolérable : Magic Kingdom}

Dès les années 2000 au Maroc, le street-art connaît un essor remarquable. La constitution et la structuration progressive de la scène festivalière marocaine, faisant la part belle aux cultures dites alternatives et aux arts de rue, y jouent un rôle 
prépondérant. À tel point que dès 2006, des observateurs enthousiastes qualifient cette effervescence culturelle et artistique urbaine de "Nayḍa ${ }^{1}$. Signifiant en arabe marocain (dārija) «le fait de se lever ", "ça bouge ", " c'est la fête », la Nayḍ marque un «moment totalement inédit au Maroc, où la parole se libérait, où la création se développait tous azimuts dans les arts urbains" (entretien avec Dominique Caubet, 2015). Les festivals, à l'instar du L'Boulevard, particulièrement emblématique de la Nayḍ $a$, se pensent et se vivent peu à peu comme des espaces d'utopies, de "construire autrement»: de nouvelles sociabilités, de nouvelles temporalités, de nouvelles structures naissent sous l'application conjointe d'une multitude d'individus, transcendant les classes sociales et les générations. Les arts de rue au Maroc, dont les festivals constituent la matrice et le noyau dur, ne s'inscrivent pas dans une confrontation directe à l'État, au Makhzen ou plus largement au modèle politico-culturel marocain. Ils constituent néanmoins des stratégies de contournement de l'autorité politique et des normes sociales qui en découlent, en utilisant notamment Internet pour diffuser leurs œuvres et leurs événements, et court-circuiter les médiateurs habituels de l'art. Ils semblent donc, au moins dans un premier temps, constituer des stratégies élargissant le spectre de réponses des acteurs subordonnés face aux dispositifs de pouvoir.

L'expansion et la structuration d'une scène urbaine prolixe et alternative, et l'énergie polémique qui en découle, sont donc observées avec une attention inquiète par certains agents du système politico-administratif marocain. Le festival constitue un espace symbolique au cœur de multiples enjeux de pouvoir, paradigmatique des dispositifs de gestion de la protestation par l'appareil étatique makhzénien. De multiples tentatives de contrôle et de domestication de la dynamique festivalière vont se développer, visant toutes à garantir, d'une manière ou d'une autre, sa mise au pas en affaiblissant et canalisant sa portée contestataire. Le contrôle de la production culturelle s'inscrit alors à travers des dispositifs plus ou moins discrets, parfois difficilement repérables, passant notamment par des tentatives d'intimidation, l'asséchement publicitaire ${ }^{2}$, l'interdiction d'événements, la cooptation et la récupération de ses figures clés. Il s'agit donc de ne pas se laisser tromper par ce que Pommerolle et Vairel qualifient de "régime de tolérance relâchée » qui se caractériserait par un «mélange d'arbitraire, de flou dans les règles et de laisser faire bonhomme " (2009:6) : l'imprédicabilité des sanctions contribue au contraire à un qui-vive permanent de la population et à un haut degré d'autocontrôle et d'autocensure des artistes et militants politico-culturels. Le contexte marocain se caractérise donc, en dépit d'une transformation formelle des modes d'exercice du pouvoir, par une continuité entre la conception hassanienne du pouvoir et le règne de Mohammed VI. Ainsi, pour Béatrice Hibou, «le politique continue largement, comme par le passé, à être désamorcé ", notamment par le biais d'un "affinement des mécanismes de cooptation, des dispositifs de maîtrise politique et économique, des technologies de domination et d'inclusion » $(2011: 6)$.

7 Les arts urbains et leur composante festivalière pourraient même constituer un espace de décompression et d'expression stratégique voire nécessaire à la pérennité du pouvoir. Ainsi,

Non seulement la contestation [produite par les pratiques artistiques urbaines] peut être tolérée par le régime, mais en plus elle s'avère fonctionnelle car elle constitue une soupape de sûreté [...], elle autorise une respiration, mais dans les limites qui lui sont imposées par le pouvoir lui-même, dont la tolérance peut s'arrêter du jour 
au lendemain si le monarque estime que ces limites ont été franchies (entretien avec René Otayek, 2015).

culturel et symbolique par l'imaginaire et la référence, par la suggestion et l'adhésion. Incorporé dans un dispositif complexe de séduction diplomatique, le festival de streetart agirait en faire-valoir de la modernité et de l'ouverture du pays à l'international, rendant ainsi moins hommage à la créativité des artistes qu'à la bienveillance de ceux qui l'ont autorisée. L'étude du street-art à travers l'espace festivalier met en lumière une des spécificités de l'anatomie de la domination politique marocaine : l'incorporation des résistances à la rhétorique hégémonique, qui permet au pouvoir de se maintenir en place, voire de se renforcer, malgré les velléités dissidentes - et parfois grâce à elles. Dès lors, la scène artistique urbaine semble-t-elle subir le même procédé que celui qui coupa l'herbe sous le pied des militants du M20F, à savoir le ressaisissement de la critique opposée au pouvoir par le pouvoir.

Palais se pare en effet de l'aura "jeuniste » et moderne du street-art. À la lisière entre art subversif et subversion de l'art, le tournant du street-art résiderait, aux yeux de Christophe Genin, dans le passage de «la contestation de l'ordre établi à son illustration » (2013: 20). Ainsi récupérés, les street-artistes deviennent, bien souvent à leur insu, les metteurs en scène du pouvoir politique, tandis que les œuvres composent sagement une galerie à ciel ouvert et transforment la ville en " manifeste d'un prince » (2013 : 164) - et, en l'occurrence, d'un roi. C'est ainsi qu'après le rendez-vous manqué $\mathrm{du}$ M20F, le street-art entre dans une phase de routinisation et de mise au pas évidente. Il va être pleinement et ouvertement intégré aux stratégies urbanistiques des grands centres urbains marocains. Cette domestication radicale vise une fin toute définie: l'identité visuelle des villes marocaines. L'immense fresque réalisée par le crew Placebo sur le boulevard Massira, comprenant le mot «Casablanca » et émaillée de références folkloriques (guerrab emblématique de la Place Jemaa El Fna, Oum Kalthoum) en est une parfaite illustration. L'œuvre agit comme un logo de la ville et marque durablement les imaginaires de ses habitants et de ses touristes. Suite au festival Remp'Arts en 2013, la petite ville d'Azemmour, coincée dans le giron d'El Jadida, est par ailleurs aujourd'hui particulièrement connue et reconnue pour la profusion d'œuvres de street-art des murs de sa médina, qui passait jusque-là sensiblement inaperçue. Le street-art devient dès lors une ressource stratégique des villes dans leurs efforts de communication et de marketing urbain. Ces scénographies relèvent, pour Jean-Pierre Garnier, d'un processus de « disneylandisation » de l'espace public (2008 : 69). Celui-ci se fait vitrine d'une villemarchandise qu'il s'agirait dorénavant selon la novlangue néolibérale de « requalifier », de « revaloriser» et de «repositionner ». Pour l'auteur, le dessein qui inspire ces efforts est immuable: «gommer les traces de la précarisation, la paupérisation et la marginalisation de masse, en mettant en scène la vision irénique d'une communauté de citadins (ré)unifiés sous le signe d'une urbanité partagée ». Un peu de poudre aux yeux pour réactiver un « mythe du bonheur collectif réactualisé sur le mode esthétique dans une sorte de Disney City, un vrai-faux magic kingdom urbain » (2008 : 75-76). 


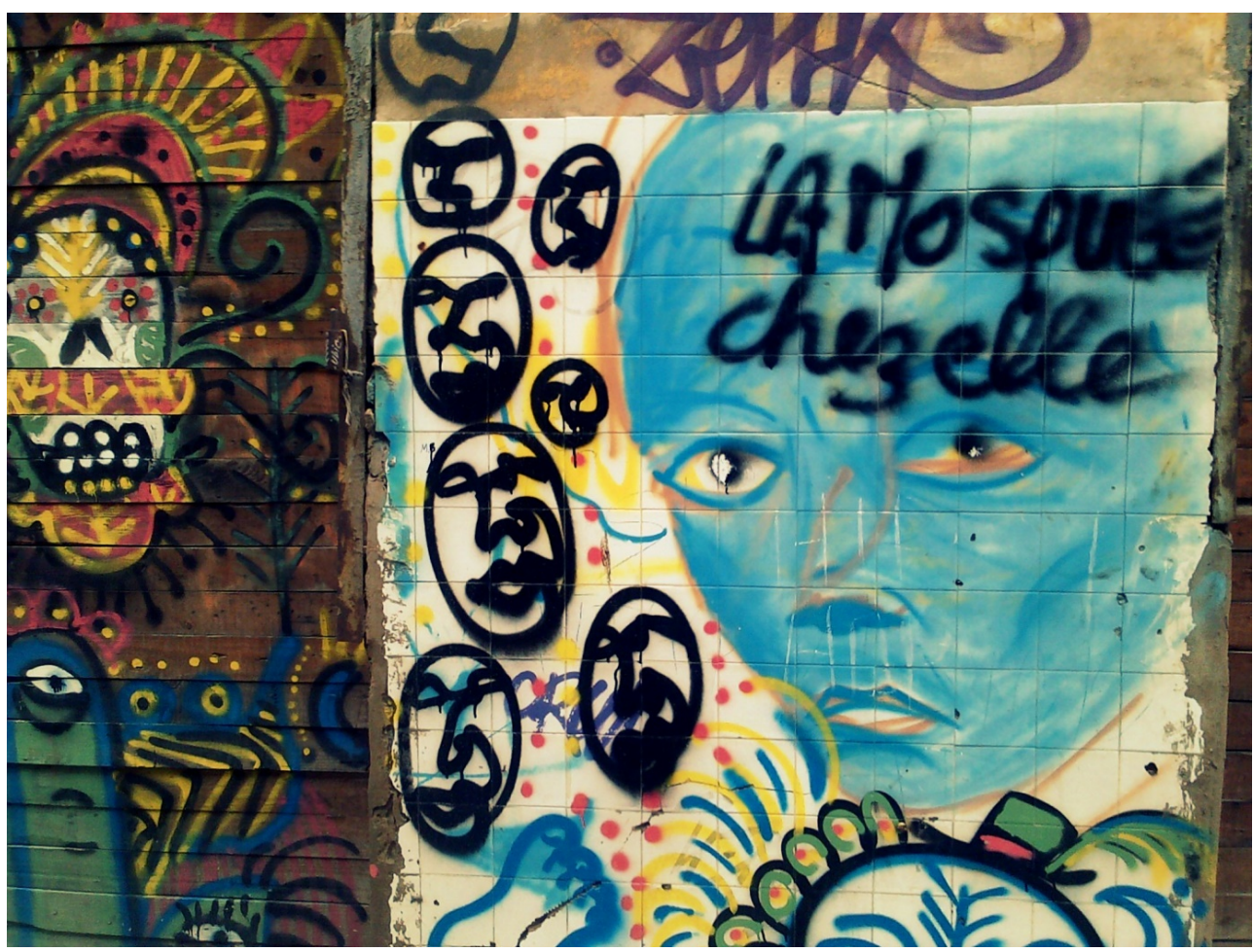

(c) Cléo Marmié

\section{Frontières du légitime : de la marge au mainstream}

11 Parallèlement à sa consécration dans des espaces festivaliers, le street-art marocain emprunte le chemin du street-art international en épousant les dynamiques et les injonctions du marché de l'art, et en faisant notamment une intrusion remarquable dans les galeries d'art et les musées marocains. La BCK Art Gallery et la David Bloch Gallery à Marrakech, la Galerie 38 à Casablanca ou encore le très institutionnel musée Mohammed VI de Rabat ont ainsi accueilli d'ambitieuses expositions de pièces de streetart entre leurs murs - troublant encore un peu plus les frontières entre art dans la rue et art de rue. L'encensement de cette pratique jusque-là désignée comme l'expression typique des exclus, témoigne d'une profonde renégociation de la charge symbolique du street-art et de ses perceptions. Ce parcours de reconnaissance offre donc une légitimité nouvelle au street-art en lui permettant de se dégager du registre de la déviance pour entrer de plein pied dans le registre de l'art, et notamment de l'art contemporain. Mais cette homologation artistique n'annule pas la suspicion qui plane sur le street-art: de la souillure du tag sur un mur à l'œuvre à protéger, où tracer la frontière ? Les ambitions de hiérarchisation et de classification des arts de rue qui mettent en avant un principe d'acceptabilité reconnaissant à telle expression une forme artistique ou non, interrogent l'ambiguïté de la distinction arbitraire "art» versus "vandalisme». L'ambiguïté du passage du street-art d'un art « vandale » à un art «vendable » se lit de manière privilégiée sur le territoire marocain, puisque cette conquête de légitimité s'y est exercée de manière particulièrement fulgurante : ayant " pris le train en marche ", il a épousé les dynamiques du marché de l'art, où la demande d'art urbain était déjà vive, presque instantanément. 
12 Dès lors, le graffiti devenant à la fois carrière et promesse lucrative, une course au spectaculaire et aux superlatifs semble s'être enclenchée: les œuvres, pour être reconnues par un public toujours plus vaste et devenir "virales ", notamment via les réseaux sociaux, semblent devoir être toujours plus grandes, étonnantes, magistrales. Les artistes ont donc de moins en moins recours à l'illégalité pour réaliser ces œuvres et sont tendanciellement plus enclins, afin de s'assurer des conditions de réalisation satisfaisantes, à privilégier les festivals ou les commandes, avec tout ce que cela implique de censure ${ }^{3}$ et d'autocensure. Et c'est bien là l'autre conséquence de ce phénomène: la volonté de toucher une "cible mondiale ", qui caractérise la culture mainstream, n'est pas sans impacter le potentiel subversif et contestataire du street-art. Renvoyant au consommateur un imaginaire jeune, révolté et "tendance", et donc désirable, le monde du graffiti devient un argument marketing comme un autre tandis que le street-art se voit transfiguré en bien de consommation et de divertissement. En témoignent par exemple la communication de Maserati pour le lancement de son showroom à Casablanca à travers le vernissage d'œuvres du graffeur Morran Ben Lahcen ou encore la prestigieuse joaillerie Bulgari qui a confié la décoration de sa boutique au crew marocain Placebo.

13 En juillet 2017, c'est la banque Umnia du groupe CIH Bank qui a illustré sa compréhension de l'intérêt stratégique du street-art pour son image de marque. Après avoir recouvert d'une affiche publicitaire l'œuvre de Hendrick Beikrich réalisée à Marrakech, la banque s'est attirée les foudres des internautes qui ont immédiatement relayé les images du forfait sur les réseaux sociaux et appelé au boycott de la banque. Umnia a rapidement présenté ses plus plates excuses sur Twitter et Facebook:

Pour votre information, ce mur est payé par le $\mathrm{CIH}$ au Syndic [sic] et offert à la Ville de Marrakech pour accueillir cette œuvre depuis sa conception. Nous y tenons comme tous les amateurs de l'art et comme tous les Marrakchis. Il s'agit d'une regrettable erreur. Merci pour votre vigilance ${ }^{4}$.

14 Attestant par ce message du caractère emblématique de l'œuvre dans l'identité visuelle de la Ville Rouge et de la légitimité du street-art dans le champ de l'art, et mesurant l'erreur stratégique qu'elle vient de commettre, Umnia Bank cède donc à la mobilisation des internautes et retire l'affiche publicitaire dans la journée. Ce dénouement n'est pas sans provoquer quelques commentaires acides sur Facebook: «Le bad buzz, l'objectif est réalisé, le Maroc tout entier connaît à présent Umnia Bank ${ }^{5}$ ».

De la marge au mainstream, il semble donc s'opérer un renversement paradoxal: la critique du système portée par le street-art n'aurait constitué qu'un moteur parmi d'autres du libéralisme économique et de la culture mainstream, qui a su se ressaisir des valeurs de liberté et d'individualité du graffiti pour les constituer en valeurs néolibérales. Ces dévoiements successifs, cette " révolte consommée » (Heath \& Potter, 2005) sont le résultat de ce que Christophe Genin qualifie d'ironie de l'histoire du streetart: ce qui était à l'origine un "cri de révolte devient alors un signe extérieur de modernité exploité par les puissances politiques, institutionnelles ou marchandes » (Genin, $2013: 20$ ). Or l'art, et a fortiori le street-art, y compris s'il ne se revendique pas " engagé ", contient dans ses principes et ses modalités d'exécution, en donnant à voir d'autres façons d'appréhender la réalité, une ambition implicite de mise en question de l'ordre social, culturel, économique ou politique. Pourtant, les revendications de subversion, d'émancipation et de transgression sont devenues peu à peu les « positions standards » des artistes contemporains et urbains. Les œuvres perdent ainsi progressivement leur virulence et donnent lieu à un désamorçage de leur contenu 
contestataire. Chaque transgression étant in fine intégrée et acceptée à la fois par les médiateurs de l'art, les institutions et le public, elle en appelle immédiatement une autre, qui sera à son tour remplacée. Jouant le jeu du marché de l'art sous prétexte de le court-circuiter, le street-art finirait par se conformer aux logiques marchandes. L'injonction toujours renouvelée à la transgression dépossède celle-ci de sa force subversive, et la dimension ludique du street-art finit par absorber totalement ses dimensions tragiques et contestataires. La marginalité devient un gage de distinction sociale, dans un anticonformisme finalement très conventionnel au regard des canons esthétiques contemporains.

En creux de l'étude du street-art au Maroc, se dessine donc un foisonnement parfois paradoxal de pratiques et de positionnements des artistes, donnant à voir une réalité complexe, faite d'agencements, de superpositions et d'enchevêtrement des intentionnalités des artistes, de détournements et de récupérations. Loin de constituer un spectre aux extrémités figées et irréconciliables, avec d'un côté le graff noble et de l'autre le tag trouble, d'un côté l'artiste de rue et de l'autre l'artiste de galerie, la vaste constellation du street-art au Maroc puise au contraire sa force et son énergie créatrice dans l'extrême mobilité de ses frontières et de ses contours. Veillons donc à ne pas figer un phénomène mouvant, mais à en souligner au contraire les jeux d'emprunts et le caractère transgénérationnel et interclasse du street-art au Maroc. Pour les artistes, la frontière se situe moins entre le tag et le graffiti qu'entre la sphère économique permettant de dégager un revenu stable et/ou une visibilité élargie à travers des réalisations sur des murs autorisés ou dans des lieux dédiés, et la sphère informelle, plus expérimentale, parfois illégale. Ils se refusent à l'injonction communément faite aux artistes urbains de choisir artificiellement entre la rue et l'institution, et se tiennent à la lisière de chacune. Ainsi, le street-art puise sa force de cette position fragile et insaisissable: en se tenant perpétuellement sur le seuil, sur la frontière. Frontière entre le permis et l'interdit, entre le légitime et l'indésirable, entre culture élitiste et art populaire, entre compromis et compromission. À la frontière également entre la rue et la galerie, s'épanouissant tour à tour d'un côté du mur ou de l'autre, dans une tension continue et souvent conflictuelle. C'est au cœur de cette capacité à jouer sur les deux tableaux que se loge la possibilité de subversion des rapports entre culture dominante et culture dominée, entre art populaire et art légitime. En déclinant le choix, les street-artistes s'assurent d'ailleurs de nouveaux leviers de pouvoirs. En brouillant les pistes entre le légitime et le délictueux, ils jouent le jeu du système pour mieux le subvertir. L'adoption de codes et de normes propres à la culture élitiste et l'insertion dans les circuits du marché de l'art, tout en proposant également des médiums, messages et créations polémiques, permet de bloquer la coercition exercée sur le street-art par sa reconnaissance et son institutionnalisation. Ce jeu permet également de piéger les autorités en rendant floue la limite entre acte vandale et production à protéger. L'ambiguïté du street-art au Maroc et ses contradictions hâtivement décriées, loin de constituer une faiblesse, constituent donc peut-être au contraire son moteur privilégié. 


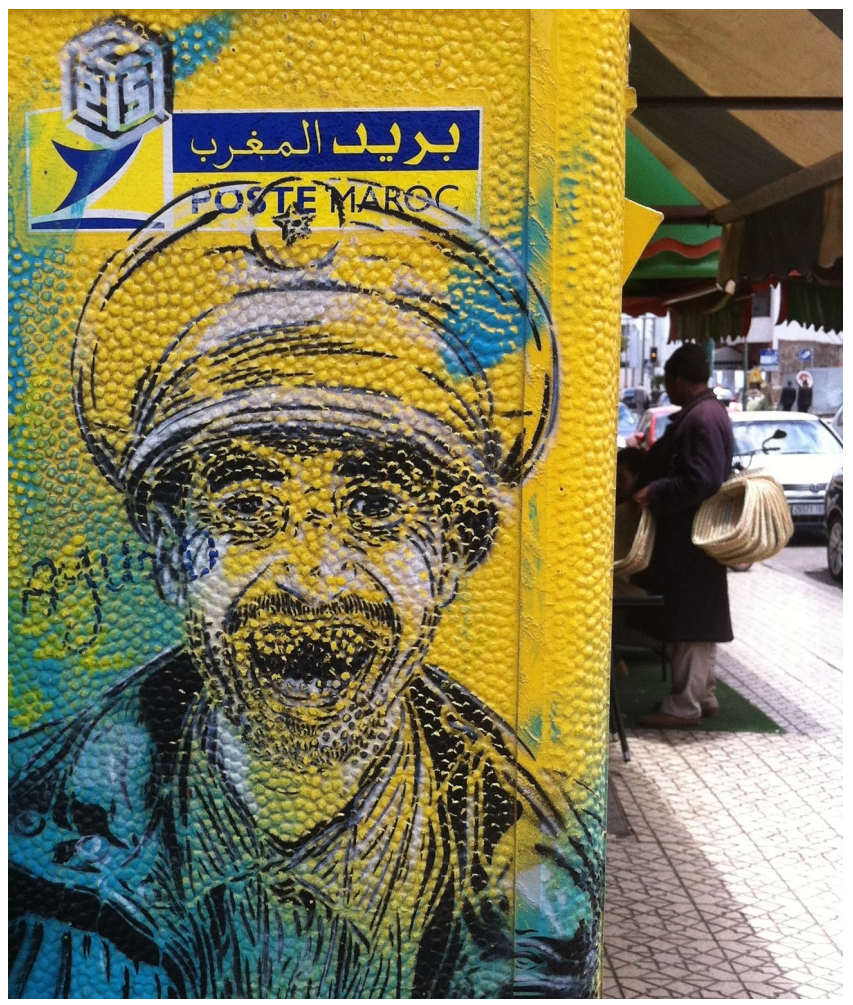

(c) Cléo Marmié

\section{Frontières du colonial : « Berrechid n'est pas le Bronx »}

Les pratiques artistiques dans l'espace public, et donc le fait d'apposer sa marque sur son environnement, sont des formes d'expression culturelles anciennes: des illustrations sur pierre ont pu être retrouvées à Pompéi, ainsi qu'à l'Agora d'Athènes ou dans la Vallée des Rois en Égypte. Il est néanmoins communément accepté que la naissance du graffiti en tant que tel date des années 1960 et 1970 aux États-Unis (Beuscart \& Lafargue, 2003: 47), pour prendre ensuite de l'ampleur et atteindre l'Europe, notamment l'Allemagne et le Royaume-Uni, puis s'élargir aux pays dits du Sud. Une première lecture de la chronologie du street-art international présume ainsi de l'importation et de l'hybridation d'une pratique culturelle et artistique occidentale dans d'autres terrains, notamment au Maghreb. Cette lecture partielle repose sur le modèle de la percée et de la dissémination.

Aux yeux de Christophe Genin, elle accrédite de façon caricaturale le processus d'américanisation des cultures, en creux duquel le graffiti, figé dans une définition états-unienne universelle, se serait propagé tel «un vent de liberté qui souffle et s'étend dans le monde" (2013: 13). La généalogie du street-art, relativement peu explorée par la littérature sociologique, pose pourtant la question des rapports d'influence et de pouvoir qui se jouent dans la thèse d'une genèse américano-centrée du graffiti. Celle-ci occulte la discontinuité et la diachronie de l'histoire du graffiti et du street-art, ainsi que la spécificité des rapports que chaque pays entretient à la rue, étroitement liée à son histoire politique, sociale et juridique. Réintroduire de la complexité dans la genèse du street-art, c'est donc permettre de rompre avec une narration fantasmée constitutive du soft power américain et de son hégémonie 
« douce ». Bien que le street-art international soit indéniablement inspiré et influencé par les pays du Nord, «Berrechid n'est pas le Bronx » (Ed Oner, in Boushaba, 2017) et le street-art au Maroc n'est pas un simulacre de l'imagerie urbaine occidentale émaillé de références folkloriques. Il est au contraire le produit d'un processus d'importation, d'hybridation et d'emprunts, puisant notamment ses influences dans le muralisme latino-américain ou encore dans la calligraphie arabe, particulièrement bien illustrée par Pascal Zoghbi et Don Karl dans leur ouvrage Le Graffiti arabe (2012).

19 Néanmoins, au Maroc comme dans d'autres pays dits du Sud, l'Occident perdure comme étalon de référence. L'incorporation du street-art à la stratégie de soft power du Maroc répond notamment à cette logique, puisqu'implicitement, c'est aussi à un dispositif de séduction de l'Occident que répond la reconnaissance du street-art par les autorités marocaines, qui y perçoivent une opportunité à la fois diplomatique et économique. Par ailleurs, en dépit des efforts des organisateurs, les artistes étrangers demeurent surreprésentés dans les manifestations de street-art de grande envergure dans le royaume, à l'instar de Sbagha Bagha à Casablanca ou de Jidar à Rabat. Le cas de la revue marocaine A+E Architecture et Environnement est également révélateur : dans le dossier du mois d'avril 2016 intitulé «L'art urbain au Maroc », sur cinq interviews d'artistes, un seul donnait la parole à un artiste marocain. De même, le Jardin Rouge, résidence d'artistes basée à Marrakech et consacrée à l'art urbain, n'a ouvert ses portes au travail de street-artistes marocains qu'en 2016. L'artiste marocaine C. B. ${ }^{6}$ témoigne quant à elle de l'implicite néocolonial de certaines pratiques artistiques dans l'espace public marocain, à travers l'exemple du Billboard Festival où des affiches publicitaires ont été investies par des œuvres dans les rues de Casablanca en 2015 à l'initiative d'une artiste danoise. C. B. regrette le procédé par lequel l'artiste étrangère est parvenue à investir l'espace public, à savoir l'achat des espaces publicitaires, qui à ses yeux a décrédibilisé le travail des artistes urbains marocains :

L'artiste elle débarque et puis elle dit « Voilà je suis danoise, j'arrive au Maroc et je parviens à investir l'espace public alors que y'a des artistes d'ici qui n'y arrivent pas depuis plusieurs années. Vous voyez c'est très facile !» [...] Quand le New York Times communique dessus en disant que l'artiste danoise a pu investir l'espace public à Casablanca... Non! C'est avec de l'argent [...] qu'elle a pu faire ça. Elle a le statut d'un annonceur. Tu achètes un panneau pour une durée déterminée, tu mets ce que tu veux dedans! Personne ne va rien te dire (entretien avec C. B., 2018).

Par ailleurs, si les œuvres de street-art marocain mettent en lumière l'intime imbrication de multiples cultures (maghrébines, européennes, américaines), la narration exalte les référentiels marocains - parfois sous des clins d'œil convenus : thé à la menthe, tajine, dromadaire et autres éléments de carte postale, d'ailleurs particulièrement usités par les artistes étrangers intervenant au Maroc. Et les artistes marocains n'échappent pas à l'écueil fréquent des artistes périphériques: créer un univers exotique afin de plaire au public international et implicitement "perpétuer une esthétique ethnographique qui laisse lire la supériorité d'une manière d'être au monde sur une autre" (Mebarki, 2011: 88). Se lit ainsi à certains égards dans les œuvres le récit d'une éternelle redevabilité, celle du graffiti arabe aux tags du Bronx, des street-artistes marocains aux writters occidentaux, et peut-être ainsi du Sud au Nord. Mais cette redevabilité contient toujours en son sein la reconnaissance implicite de la supériorité du premier sur le second, qui se permet d'emprunter sans jamais s'y confondre. Les mécanismes esthétiques, graphiques, commerciaux et argumentatifs 
propres au monde de l'art en Occident persistent dès lors en tant que forces légitimatrices de l'art et de l'art urbain au Maroc.

Ainsi, le street-art au Maroc est révélateur de la porosité des cultures et des rapports de pouvoir qui le traversent. S'inscrivant indéniablement dans un contexte d'inspiration réciproque mais inégal avec l'Occident, il illustre des relations complexes et pluridirectionnelles d'opposition, de complémentarité, de rejet voire de subordination d'une culture à l'autre, d'une culture sur l'autre. Ces arts visuels interrogent les relations Nord-Sud, et fécondent une réflexion postcoloniale. "Affiches, graffitis, stencils, autocollants, tags et autres formes expressives témoignent de l'appropriation voire de la sémiotisation des lieux de passage » d'une culture à l'autre (Botero, $2011: 2$ ), et éclairent sur la richesse, la complexité et les écueils de l'interculturalité et de la mondialisation de l'art. Pour reprendre la terminologie de Pascale Casanova à propos de l'univers littéraire mondial et en le transposant plus généralement à l'univers culturel et artistique, le street-art marocain tend donc à jouer le jeu du marché de l'art international afin de transcender sa position d'« excentrique ", de marginal. Il fait alors écho à la saisissante réplique de Khatibi : "Quand je danse devant toi, Occident, sans me dessaisir de mon peuple, sache que cette danse est de désir mortel » $(1971: 188)$. En donnant à voir un cadre national périphérique, et en affirmant la légitimité artistique d'une voix excentrée, le street-art marocain subvertit les codes, les règles et le langage $\mathrm{du}$ centre artistique mondial, après en avoir justement usé pour accéder à la reconnaissance. Dès lors, selon les schèmes de catégorisations de Pascale Casanova, bien plutôt qu'assimilés, les artistes urbains marocains se révèlent bien plutôt révolutionnaires, capables de produire des innovations formelles et esthétiques extraordinaires (Casanova, 1999).

Le calli-graffiti, issu de la tradition calligraphique arabe et du street-art, en est un exemple particulièrement riche. Le street-art au Maroc recèle d'exemples du mariage du writting et de la calligraphie arabe et d'artistes ayant réalisé cette convergence. Celle-ci ne se lit pas uniquement dans des œuvres réalisées dans la rue ou exposées dans des espaces culturels, mais se lit également dans la vie quotidienne de l'espace public marocain, à l'instar des enseignes calligraphiées des boutiques de fortune ou encore des inscriptions colorées des camions. Plus encore, le lightgraff marocain prolonge la rencontre féconde entre calligraphie et street-art en l'appliquant à la "peinture à la lumière " (lightpainting), technique reposant sur la prise de vue photographique de traits de lumière. Cette discipline hybride et innovante d'écriture dans l'espace, représentée au Maroc notamment par le collectif marrakchi KechLight, se situe à la croisée du langage corporel, du calli-graffiti et de la photographie.

L'une des propriétés fondamentales du street-art arabe en général et marocain en particulier réside donc dans la convocation et la projection multidirectionnelle d'images, d'intentions et de symboles autoréférencés, métis ou adoptés. En ce sens, il s'inscrit dans la créolisation du monde, ce processus imprévisible cher à Édouard Glissant par lequel la mise en contact d'éléments culturels hétérogènes parvient à devenir relation et à faire éclore une nouvelle cohérence, qui ne serait ni synthèse ni addition. Cet inépuisable créatif est à la fois un médium de mise en contact et un enjeu de pouvoir, mêlant images populaires et récits d'élites, dans le rythme de son imprévisibilité et par l'ouvrage patient de ses artistes. D'importants efforts sont ainsi engagés pour renforcer et entériner les relations Sud-Sud en matière d'art contemporain en général et de street-art en particulier. En témoignent notamment la 
programmation dédiée à l'Afrique de l'Ouest du festival Awaln'Art (Rencontres Artistiques en Places Publiques) de Marrakech en 2016, le projet curatorial résolument postcolonial de la Marrakech Biennale et de son volet «MB6 Street Art » ou encore l'invitation croissante de street-artistes du Sud et notamment latino-américains, dans les festivals emblématiques du street-art au Maroc, à l'instar de Sbagha Bagha ou de Jidar.

\section{Conclusion - Un art sous tension}

Le street-art au Maroc se révèle donc un art de conflictualité, traversé de rapports de force dont les enjeux de pouvoir sont en perpétuelle renégociation. Se tenant à la lisière du tolérable, du légitime et du colonial, le street-art, au Maroc comme ailleurs, se caractérise par l'oscillation incessante qu'il opère entre pratique subversive et pratique subvertie, entre art vandale et art vendu, entre revendication postcoloniale et praxis dominée - sans que rien ne laisse présager de ce qui l'emportera sur l'autre. Le streetart est donc un art en train de se faire : un art sous tension. Et c'est justement dans cette capacité à jouer sur plusieurs tableaux et à décliner l'injonction au choix que les artistes s'assurent de nouveaux leviers de pouvoir : jouer le jeu du système pour mieux le subvertir en faisant cohabiter des pratiques disloquées, des positions mouvantes, des médiums non prédéfinis qui interdisent les catégories et suspendent le jugement de ses détracteurs comme de ses partisans. Étudier le street-art tel qu'il s'exprime au Maroc, c'est ainsi explorer les seuils, la perméabilité et les relations des sphères artistiques, esthétiques et politiques. Son étude prouve que les artistes urbains marocains, en dépit $\mathrm{du}$ faible contenu contestataire apparent de leurs œuvres, ne sont pas moins engagés qu'ailleurs, léthargiques ou mutiques, mais pris dans un réseau de contraintes spécifiques. Ainsi, comment reprocher la marchandisation spectaculairement rapide du street-art au Maroc et la faible intentionnalité politique de ses artisans alors que celui-ci s'épanouit à un moment historique de reconnaissance du street-art par le marché de l'art contemporain international et que sa pratique illégale au Maroc exige un « coût de l'engagement » (Vairel, 2014: 19) particulièrement élevé ? En s'inspirant de la pensée de CharlesBonn sur le roman maghrébin, il est ainsi à regretter que «la communication avec certains publics s'installe dans un malentendu entretenu par la complémentarité entre lecture exotique et lecture militante » (Bonn, 1997 : 184), qui l'une et l'autre passent à côté de l'activité majeure du graffeur : l'art. On comprend dès lors aisément que la pratique du street-art puisse revêtir au Maroc la forme d'une pratique ludique, festive, plus « visuelle » que "militante », agissant comme un contournement en acte des normes du pouvoir, une distanciation de «la» politique. Mais cette désertion de la sphère politicienne ne signifie pas pour autant une désertion « du » politique.

L'étude du street-art s'inscrit ainsi sur la piste du politique "ailleurs », en invitant à porter «le regard en dehors des sites les plus évidents d'observation politique " (Banégas, $2010: 2$ ), pour appréhender plus largement l'anatomie du dissentiment au Maroc, en se saisissant des espaces et des micro-pratiques de résistance, en interrogeant leurs visées insurrectionnelles, y compris les plus discrètes, et les processus de routinisation et d'instrumentalisation dont elles font l'objet. 


\section{BIBLIOGRAPHIE}

BANÉGAS Richard, SimÉANT Johanna, POMMERolle Marie-Emmanuelle (2010), « Lutter dans les Afriques ", Genèses (81).

BeusCART Jean-Samuel \& LAFARGUE DE GRANGENEUVE Loïc (2003), « Comprendre le graffiti à New York et à Ivry (Note liminaire aux textes de Richard Lachmann et de Frédéric Vagneron) », Terrains \& travaux, 5(2).

BONN Charles \& GARNIER Xavier [dir.] (1997), Littérature francophone. Tome 1 : Le Roman, Paris : Hatier.

BoUBiA Amina (2014), Les nouvelles formes de production du politique dans le monde arabe à l'exemple des festivals de musique au Maroc: culture et politique en contexte autoritaire (thèse de doctorat), Institut d'études politiques Paris, en co-tutelle avec l'Université de Cassel (Allemagne).

BOTERo Nataly (2011), « La stéréotypie Nord/Sud dans le street art latino-américain », Amerika (4), <http://journals.openedition.org/amerika/2235>.

BousHABA Jamal (2017), « Petites histoires de street-art marocain », Tel quel (863), <http:// telquel.ma/2017/05/13/petites-histoires-street-art -marocain_1546672>.

CASANova Pascale (1999), La République mondiale des lettres, Paris : Seuil.

GARNIER Jean-Pierre (2008), « Scénographies pour un simulacre : l'espace public réenchanté », Espaces et sociétés (134), <https://www.cairn.info/revue-espaces-et-societes-2008-3-page-67.htm>. GENIN Christophe (2013), Le street-art au tournant. Reconnaissance d'un genre, Bruxelles : Éd. Les Impressions nouvelles.

HEATH Joseph \& POTTER Andrew (2005), Révolte consommée. Le mythe de la contre-culture, Paris : Naïve.

HівоU Béatrice (2011), Le mouvement du 20 février, le Makzen et l'antipolitique. L'impensé des réformes au Maroc (Dossiers du CERI), Sciences Po Paris, <https://www.sciencespo.fr/ceri/sites/ sciencespo.fr.ceri/files/art_bh2.pdf>.

KHATIBI Abdelkébir, (1971), La Mémoire tatouée, Paris : Denoël.

MEBARKI Belkacem (2011), « Ce que le jour doit à la nuit. Père et repères », Résolang. Littérature, linguistique \& didactique (5), 87-95, <https://sites.univ-lyon2.fr/resolang/no05.php>.

MARTIN Denis-Constant (1989), « À la quête des OPNI (objets politiques non identifiés). Comment traiter l'invention du politique ? ", Revue française de science politique, $39^{e}$ année (6).

POMMERolle Marie-Emmanuelle \& VAIREL Frédéric (2009), « S'engager en situation de contrainte », Genèses, 77(4).

RANCIÈRE Jacques (2008), Le spectateur émancipé, Paris : La Fabrique édition.

VAIREL Frédéric (2014), Politique et mouvements sociaux au Maroc. La révolution désamorcée ?, Paris :

Presses de Sciences Po.

ZoGHBI Pascal \& KARL Don [dir.] (2012), Le Graffiti arabe, Paris : Eyrolles. 


\section{NOTES}

1. En arabe standard Nahda, terme très connu pour désigner la Renaissance arabe qui a eu lieu dans le monde arabe (d'abord en Égypte avant de s'étendre au reste du monde arabe) du milieu $\mathrm{XIX}^{\mathrm{e}}$ siècle aux premières décades $\mathrm{du} \mathrm{Xx}^{\mathrm{e}}$ siècle, marquant ainsi une effervescence linguistique, culturelle, littéraire, sociale, etc.

2. Les festivals, souvent gratuits, nécessitent d'importantes sources de financement, garanties par les sponsors: les grands acteurs économiques privés du Maroc (FC.com, Maroc Telecom, Sidi Ali, Inwi, OCP...), historiquement liés au Palais, constituent donc les partenaires privilégiés des festivals d'arts urbains - et l'on comprend aisément le chantage implicite que cette configuration tend à induire. Les pouvoirs économiques et politiques, intimement imbriqués jusqu'à se confondre, fixent et ordonnent ainsi implicitement les enjeux et le contenu des festivals. Et donnent le « la » de l'alternative au Maroc, jusqu'à parfois provoquer l'annulation de certains événements en suspendant leur financement.

3. C'est ainsi qu'à l'occasion de la Street-Art Caravane qui s'est tenue à Safi en mai 2016, Mouhim Simo a été contraint par les organisateurs du festival de modifier l'une de ses œuvres (représentant initialement une femme flottant au milieu d'ustensiles de cuisine) sans que l'origine de l'ordre de censure ne soit révélée - non sans provoquer l'incompréhension et l'indignation sur les réseaux sociaux. À l'occasion de Billboard Festival, c'était le partenaire afficheur FC.com (détenu par Mounir Majidi, secrétaire particulier de Mohammed VI depuis les années 2000) qui avait prié l'artiste marocaine Randa Maroufi de retirer l'une des photographies qu'elle avait prévu d'exposer dans l'espace public. La photographie, qui représente une scène de harcèlement de rue, a finalement été autorisée à l'affichage.

4. Déclaration de M. Rahhou rapportée sur la page Facebook officielle d'Umnia Bank.

5. Commentaire publié le 8 juillet 2017 sur la page Facebook de Umnia Bank suite à l'incident.

6. Afin de préserver l'anonymat de l'artiste, les initiales ont été changées.

\section{RÉSUMÉS}

Les pratiques artistiques urbaines qui composent la vaste constellation du street-art sont aujourd'hui durablement inscrites dans les rues marocaines. Ces interventions visuelles colorées, imprévisibles et mouvantes interrogent les façons d'appréhender l'espace public marocain. Se tenant à la lisière du tolérable, du légitime et du colonial, le street-art au Maroc se caractérise par l'oscillation incessante qu'il instaure entre pratique subversive et pratique subvertie, entre art vandale et art vendu, entre revendication postcoloniale et praxis dominée - sans que rien ne laisse présager de ce qui l'emportera sur l'autre. En conviant à une réflexion transdisciplinaire inspirée de la science politique, de la sociologie politique, de l'esthétique critique et des cultural studies, cette contribution propose d'explorer les modalités par lesquelles le potentiel dissensuel du street-art au Maroc s'exprime, donnant à voir la richesse, la diversité et les enjeux contemporains d'une pratique artistique urbaine en Méditerranée.

Nowadays, the urban artistic practices which make up the large constellation of street art are an undeniable part of the landscape of Moroccan cities. These colorful, visual, moving, and unpredictable interventions question the different ways of comprehending the Moroccan public space. On the edge of permissible, legitimate, and colonial, street art in Morocco is characterized 
by the constant oscillation between subversive and subverted practices, between vandal and marketed art, between postcolonial revendication and dominated praxis - without any indication on what will outweigh the other. Through transdisciplinary thinking and by combining political science, political sociology, critical aesthetic and cultural studies, this article aims to explore the conditions through which the dissensual potential of Moroccan street art exists, and how it highlights the richness, the diversity and the contemporary issues of this urban artistic practice.

\section{INDEX}

Mots-clés : Maroc, street-art, graffiti, art et politique, domination, résistance, espace public, Nord et Sud

Keywords : Morocco, street-art, graffiti, arts and politics, domination, resistance, public space, North and South

\section{AUTEUR}

\section{CLÉO MARMIÉ}

Université Paul-Valéry Montpellier 3 